Geometry and Codes 


\section{Mathematics and Its Applications (Soviet Series)}

Managing Editor:

M. HAZEWINKEL

Centre for Mathematics and Computer Science, Amsterdam, The Netherlands

Editorial Board:

A. A. KIRILLOV, MGU, Moscow, US.SR.

Yu. I. MANN, Steklov Institute of Mathematics, Moscow, USS.SR.

N. N. MOISEEV, Computing Centre, Academy of Sciences, Moscow, U.S.S.R.

S. P. NOVIKOV, Landau Institute of Theoretical Physics, Moscow, U.S.SR.

M. C. POLYVANOV, Steklov Institute of Mathematics, Moscow, U.S.S.R.

Yu. A. ROZANOV, Steklov Institute of Mathematics, Moscow, U.S.SR. 


\section{D. Goppa}

Computer Center of the Academy of Sciences of the U.S.S.R., Moscow

\section{Geometry and Codes}

Translated by N. G. Shartse

SPRINGER-SCIENCE+BUSINESS MEDIA, B.V. 
Library of Congress Cataloging in Publication Data

Goppa , V. D.

Geometry and codes.

(Mathematics and its applications ; 24. Soviet series)

Bibliography: p.

1. Error-correcting codes (Information theory)

2. Geometry. I. Title. II. Series: Mathematics and its applications (Kluwer Academic Publisher's). Soviet, series ; 24 .

QA268.G576 $1988 \quad 005.6 ' 2 \quad 88-9384$

ISBN 978-1-4020-0302-8 ISBN 978-94-015-6870-8 (eBook)

DOI 10.1007/978-94-015-6870-8

$02-0391-200$ ts

\section{All Rights Reserved}

(C) 1988 by Springer Science+Business Media Dordrecht

Originally published by Kluwer Academic Publishers in 1988

No part of the material protected by this copyright notice may be reproduced or utilized in any form or by any means, electronic or mechanical including photocopying, recording or by any information storage and retrieval system, without written permission from the copyright owner 


\section{SERIES EDITOR'S PREFACE}

Approach your problems from the right end and begin with the answers. Then one day, perhaps you will find the final question.

'The Hermit Clad in Crane Feathers' in R. van Gulik's The Chinese Maze Murders.
It isn't that they can't see the solution. It is that they can't see the problem.

G.K. Chesterton. The Scandal of Father Brown 'The point of a Pin'.

Growing specialization and diversification have brought a host of monographs and textbooks on increasingly specialized topics. However, the "tree" of knowledge of mathematics and related fields does not grow only by putting forth new branches. It also happens, quite often in fact, that branches which were thought to be completely disparate are suddenly seen to be related.

Further, the kind and level of sophistication of mathematics applied in various sciences has changed drastically in recent years: measure theory is used (non-trivially) in regional and theoretical economics; algebraic geometry interacts with physics; the Minkowsky lemma, coding theory and the structure of water meet one another in packing and covering theory; quantum fields, crystal defects and mathematical programming profit from homotopy theory; Lie algebras are relevant to filtering; and prediction and electrical engineering can use Stein spaces. And in addition to this there are such new emerging subdisciplines as "experimental mathematics", "CFD", "completely integrable systems", "chaos, synergetics and large-scale order", which are almost impossible to fit into the existing classification schemes. They draw upon widely different sections of mathematics. This programme, Mathematics and Its Applications, is devoted to new emerging (sub)disciplines and to such (new) interrelations as exempla gratia:

- a central concept which plays an important role in several different mathematical and/or scientific specialized areas;

- new applications of the results and ideas from one area of scientific endeavour into another;

- influences which the results, problems and concepts of one field of enquiry have and have had on the development of another.

The Mathematics and Its Applications programme tries to make available a careful selection of books which fit the philosophy outlined above. With such books, which are stimulating rather than definitive, intriguing rather than encyclopaedic, we hope to contribute something towards better communication among the practitioners in diversified fields.

A code is a subset of all the words, of a given length, say, which can be written in a given alphabet, say, the alphabet consisting of the symbols ' 0 ' and ' 1 '. If all code words are sufficiently different, a certain number of transmission errors can be detected. This leads to such questions as best error correcting codes for a given transmission rate and others. Other considerations involve efficiency of coding and decoding (and error correction) algorithms.

A number of years ago the author of this book discovered that the theory of algebraic curves (and their Jacobians) over finite fields can be used to construct valuable codes. These are now named Goppa codes and the subject has become an additional important chapter in the general and 
fast growing field of coding theory. This is, so far, the first book on the subject and both because of its importance and because it links algebraic geometry and coding theory it fits perfectly with the general philosophy of this series as expressed above in the general part of this preface. I am greatly pleased to be able to welcome this unique book in this series.

The unreasonable effectiveness of mathematics in science ...

\section{Eugene Wigner}

Well, if you know of a better 'ole, go to it.

Bruce Bairnsfather

What is now proved was once only ima-

gined.

William Blake

Bussum, May 1988
As long as algebra and geometry proceeded along separate paths, their advance was slow and their applications limited.

But when these sciences joined company they drew from each other fresh vitality and thenceforward marched on at a rapid pace towards perfection.

Joseph Louis Lagrange.

Michiel Hazewinkel 


\section{Table of Contents}

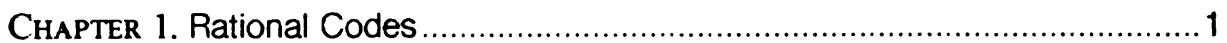

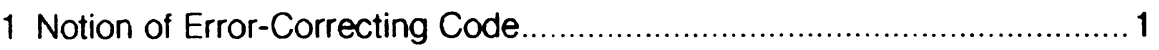

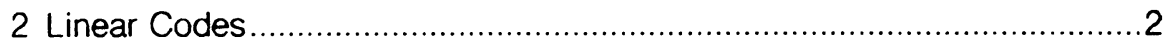

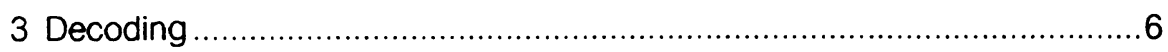

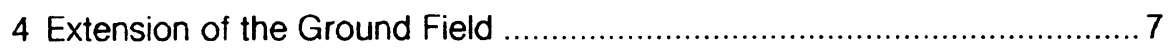

5 Hamming Code Correcting a Single Error ........................................... 9

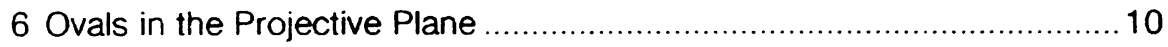

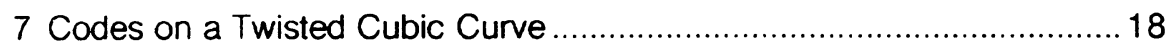

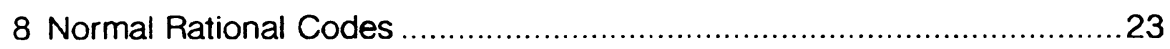

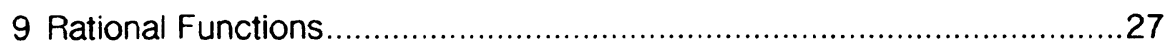

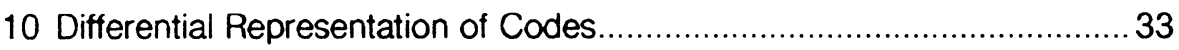

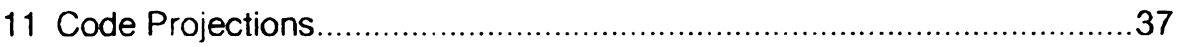

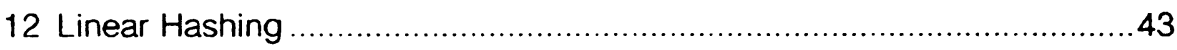

ChAPTER 2. Decoding and Rational Approximations ..................................... 47

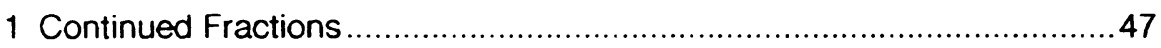

2 The Fermat Theorem on the Sum of Two Squares............................... 49

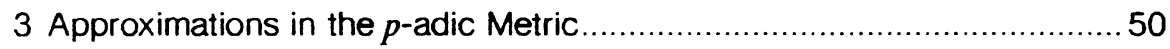

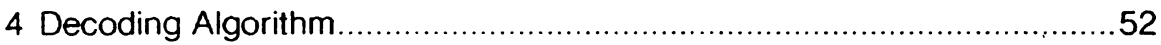

5 Linear Recurrent Sequences and Shift Registers..................................54

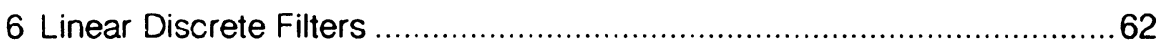

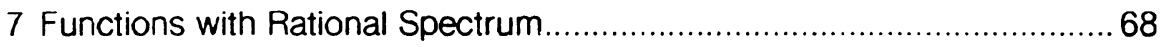

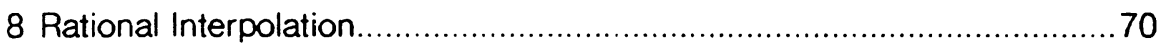

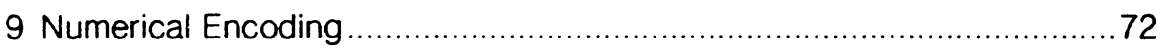

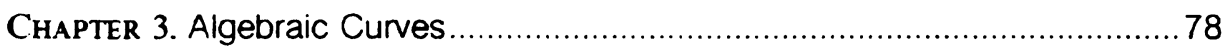

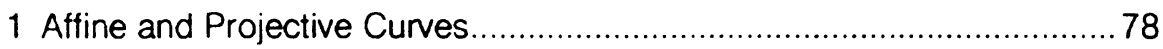


2 Intersection of Curves. Bezout Theorem.............................................. 79

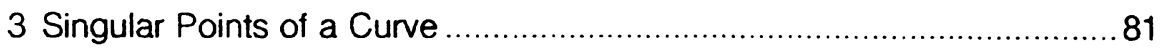

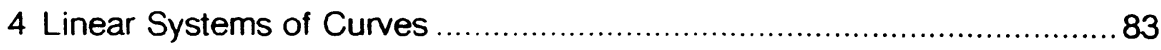

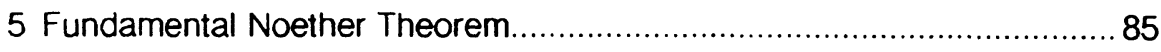

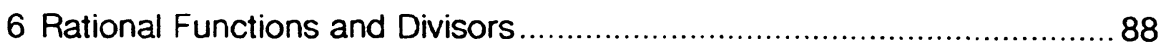

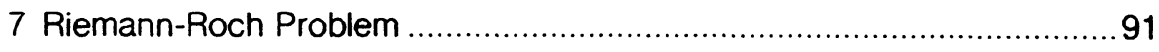

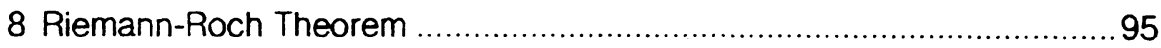

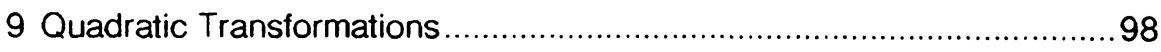

10 Projective Model of the Linear System ............................................... 102

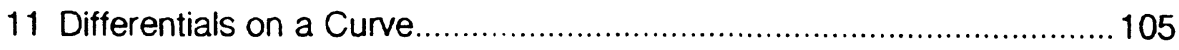

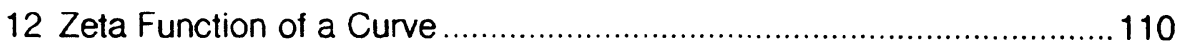

Chapter 4. Generalized Jacobian Codes ……….............................................. 115

1 The 9 -equivalence on Curves. Definition of the Code ........................ 115

2 Local Symbol. Error-Correcting Capacity of a Code ............................ 117

3 The Structure of the Error-Checking Matrix ....................................... 119

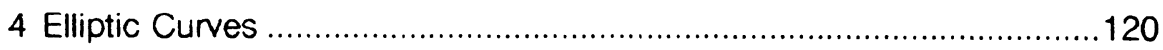

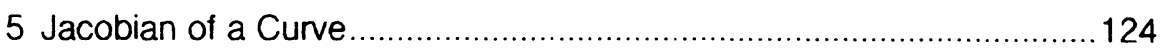

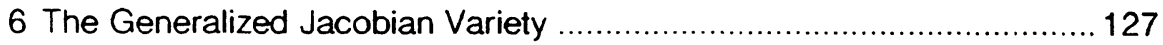

7 The Fermat and Hermite Curves ....................................................... 129

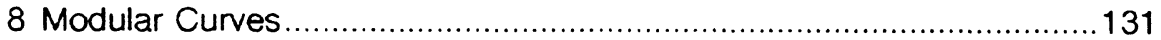

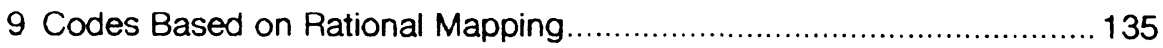

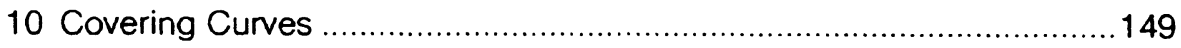

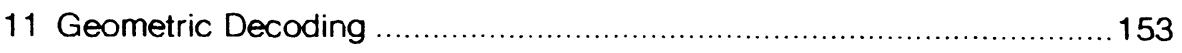

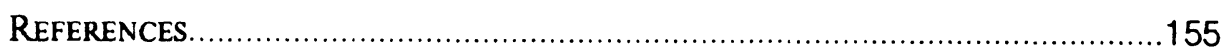

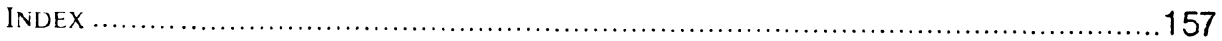




\section{Preface}

An algebraic-geometric approach to coding theory is developed in this book. Many of the concepts and results of algebraic geometry can be translated nicely into properties of error-correcting codes. Following an account of the historical development of algebraic geometry, codes are constructed. At first, these codes are constructed from conics and rational curves, and subsequently the codes are developed from linear series and the generalized Jacobian variety of a curve. Here, the Riemann-Roch theorem becomes the basic computational tool for estimating the code parameters.

The idea of applying the Riemann-Roch theorem to the analysis of codes was proposed earlier in my article "Codes, associated with divisors" (Problems of Information Transmission, 1977, No. 1, which was written on the occasion of the 25th anniversary of the fundamental Hamming article, introducing errorcorrecting codes). The present book can be considered as a realization of that idea.

The book does not presume the reader's familiarity with coding theory for algebraic geometry.

I am grateful to Professor M. Hazewinkel for his kind invitation to publish such a book in "Mathematics and Its Applications" series.

Moscow

V.D. Goppa 